\title{
ITTP-PG: A Novel Grouping Technique to Enhance VoIP Service Bandwidth Utilization
}

\author{
Mayy Al-Tahrawi ${ }^{1}$, Mosleh Abulhaj ${ }^{2}$, Yousef Alraba'nah ${ }^{3}$, Sumaya N. Al-Khatib ${ }^{4}$ \\ Department of Computer Science, Al-Ahliyya Amman University, Amman, Jordan ${ }^{1}$ \\ Department of Networks and Information Security, Al-Ahliyya Amman University, Amman, Jordan², \\ Department of Software Engineering, Al-Ahliyya Amman University, Amman, Jordan ${ }^{3}$
}

\begin{abstract}
Recently, the field of telecommunications started to migrate to Voice over Internet Protocol (VoIP) service. VoIP service applications produce packets with short payload sizes to reduce packetization delay. That is, increasing the preamble size and expends the network link bandwidth. Packet grouping is a technique to enhance the employment of network link bandwidth. Numerous grouping techniques are suggested to enhance link bandwidth employment when using RTP/UDP protocols. Unlike previous research, this article suggests a packet grouping technique that works over the Internet Telephony Transport Protocol (ITTP), not RTP/UDP. This technique is called ITTP Packet Grouping (ITTP-PG). The ITTP-PG technique groups VoIP packets, which exist in the same route, in a single ITTP/IP preamble instead of an ITTP/IP preamble to each packet. Consequently, preamble size is diminished and network link bandwidth is saved. ITTP-PG also adds 3-byte runt-preamble to each packet to distinguish the grouped packets. The suggested ITTP-PG technique is simulated and compared with the conventional ITTP protocol (without grouping) using three elements, namely, the number of concurrent VoIP calls, preamble overhead, and bandwidth usage. Based on all these elements, the ITTP-PG technique outperforms the conventional ITTP protocol. For example, the result shows that bandwidth usage improved by up to $45.9 \%$ in the tested cases.
\end{abstract}

Keywords-Voice over Internet Protocol (VoIP); Internet Telephony Transport Protocol (ITTP); packet grouping; network bandwidth

\section{INTRODUCTION}

The Internet has grown explosively in the fast few decades. This explosive growth has been accompanied by a tremendous number of new technologies such as Voice over IP (VoIP) $[1,2]$. There are quite a large number of applications that are used for making VoIP conversations, including Skype, FaceTime, and Google Hangout. The number of registered Skype users in 2017 was around 1.33 million [3,4]. Despite that, VoIP faces two main dilemmas that slows down its propagation among organizations and people. First, the low quality of the VoIP conversation, particularly compared to the typical telecommunication system. This is because VoIP infrastructure is IP-based, thus, it shared and does not provide a dedicated channel for the VoIP conversations. However, the traditional telecommunication systems provide a dedicated channel for its VoIP conversation [5,6]. Second, the failure in making the best use of network bandwidth. This is because a considerable size of VoIP packet protocols is attached to the small packet payload that is produced by the VoIP codecs $[7,8]$.
Codec (a portmanteau of coder-decoder) is a tool (hardware or software) that converts the analog voice data to digital data. The digital voice data, then, constitute the voice frame (VoIP packet payload). The Codec digitizes analog voice data after analog voice is captured for a certain period. The longer the period the bigger the voice frame size and the more the delay. The shorter the period the smaller the voice frame size and the less the delay. Considering this, and because VoIP is highly delay-sensitive, the Codec reduces the analog voice digitization period to avoid introducing an inadmissible delay. Therefore, the Codec produces small voice frames size, typically, 10 to 30 bytes based on the used codec. Table I shows some of the common VoIP Codecs [9,10,11]. As for VoIP protocols, there are two types: signaling protocols and media transfer protocols [12,13]. H.323 and Session Initiation Protocol (SIP) are the two common signaling protocols $[13,14]$. On the other hand, the 12-bytes Real-time Transport Protocol (RTP), 6-bytes Internet Telephony Transport Protocol (ITTP), and 4-bytes Inter-Asterisk eXchange (IAX) are the main examples of media transfer protocols $[9,13,15]$. Both RTP and IAX take the help of the 8-bytes User Datagram Protocol (UDP) to be able to convey the voice data, while ITTP is able to carry the voice data by itself $[9,13,15]$. As mentioned earlier, adding these protocols along with the 20-bytes IP protocol to the small VoIP packet payload leads to a considerable amount of the wasted bandwidth $[7,8]$. The wasted bandwidth is calculated by dividing the protocol size over packet size (payload + protocol). For example, the wasted bandwidth caused when using the ITTP protocol, which is our concern in this article, is up to 72.2 \% with 10 bytes codec frame size [16,17]. Fig. 1 shows the typical ITTP packet format.

TABLE I. COMMON VOIP CODES

\begin{tabular}{|l|l|l|}
\hline Code Name & Frame Size (B) & Bit Rate (kbps) \\
\hline G.723.1 & 20 & 5.3 \\
\hline G.726 & 30 & 24 \\
\hline LPC & 14 & 5.6 \\
\hline G.729 & 10 & 8 \\
\hline G.728 & 10 & 16 \\
\hline
\end{tabular}

\begin{tabular}{|c|c|c|}
\hline Payload & ITTP & IP \\
\hline 10B to 30B & $6 B$ & $20 B$ \\
\hline
\end{tabular}

Fig. 1. ITTP Packet Format. 
VoIP packets grouping is one of the key techniques to handle this problem and improve the efficiency of utilizing the network bandwidth by VoIP service [3, 18]. VoIP packets grouping techniques group several VoIP packets in one preamble, thus, saving the bandwidth. The saved bandwidth depends on the number of grouped packets in one preamble [3, 18]. This article will suggest a new VoIP packets grouping technique that groups a multiplicity of VoIP packets that travel over the same path to the same destination. Unlike the existing techniques, the suggested technique performs the grouping process at the ITTP layer, not the IP layer, which achieves better bandwidth saving.

The remainder of this article is arranged as follows: Section 2 highlights some of the key VoIP packet grouping techniques. Section 3 explains in detail the technique. It explains the location at which the suggested technique produces its best performance, the main components of the suggested method, and the internal process of the suggested method. Section 4 investigates the performance of the suggested method compared with the conventional ITTP protocol (without grouping). Finally, section 5 concludes the article.

\section{RELATED WORK}

The VoIP service media carriers protocols, in particular ITTP, groups with IP, introduce extraordinary preamble size to the VoIP data and, thus, expends the VoIP network bandwidth. VoIP packet grouping is one of the primary techniques that are used to lessen the preamble size resulting from ITTP/IP. In this section, we will highlight some of the key VoIP packet grouping techniques.

In 2012 [19], Azevêdo Filho PH et al have suggested a packet grouping technique, called Holding Time Aggregation (HTA), that works over ad hoc wireless networks. The main objective of the HTA technique is to reduce the number of transmissions and transmission overhead, by adaptively grouping several VoIP packets in one preamble. HTA technique keeps the VoIP packets for a certain time to group them. The time chosen by the HTA technique changing adaptively based on the current network conditions, in order to keep the VoIP service quality with the acceptable score. The suggested HTA technique was estimated in comparison to several other techniques, in which it outperformed all the comparable techniques. Whereas, HTA proved that it is able to reduce the number of resources needed to carry the generated traffic, both in terms of packet transmissions and the volume of bytes carried over the network. The implementation of the HTA technique has proven that it is able to maintain jitter and total delay within tolerable limits of VoIP service. In addition, the HTA technique achieves a substantial reduction in the number of packet transmissions as well as the overall packet overhead. The savings in terms of packet transmissions can be as high as $80 \%$ in the evaluated scenarios.

Another VoIP grouping technique was suggested by Nomura, Yoshihide, et al in 2014 [18]. The suggested packet grouping technique works in IEEE802.11ac wireless network. The suggested technique grouped several packets directing to the same mobile terminal into a single IEEE802.11ac wireless frame. In order to achieve high efficacy, the suggested technique takes into account the multi-rate transmissions and
VoIP service. In addition, it gives the priority of transmission to the mobile terminal that has buffered VoIP packets beyond the tolerable VoIP delay. Moreover, the space channel time is reduced by regulate the transmission times of wireless frames through adjusting the number of grouped packets considering their modulation and coding scheme (MCS) level. The suggested scheme is evaluated by computer simulation to demonstrate its effectiveness under the WLANs with multi-rate transmission providing VoIP services. The results of computer simulations show that the suggested scheme improves the system throughput, the space channel time ratio during MUMIMO transmissions, and maximum delay time for the VoIP packets in the WLANs with multi-rate transmissions. Consequently, the suggested packet grouping scheme is effective for the downlink MU-MIMO channels under recent WLAN environments.

More recently, in 2019 [20], Abualhaj M. et al have suggested a grouping technique, named compressionaggregation over ITTP (CA-ITTP), that works over the ITTP protocol. As the name suggests, the CA-ITTP aims to save the bandwidth by i) compressing the VoIP packet payload and ii) grouping several ITTP protocol VoIP packets in one preamble. The VoIP packet payload compression is achieved by finding and transmitting the difference between the successive VoIP packets payload. The ITTP protocol VoIP packets grouping is achieved grouping several VoIP packets at the IP layer. In other words, several VoIP packet payload and ITTP preamble of different packets are grouped in one preamble. In order to reconstruct the original packet at the receiver side, a minipreamble is attached to each VoIP packet payload and ITTP preamble at the sender side. The performance evaluation, of the suggested CA-ITTP technique in comparison to the traditional ITTP protocol, has shown considerable improvement of the bandwidth usage. Whereas, the number of concurrent calls that run over the same network bandwidth is almost doubled, when using the suggested CA-ITTP technique in comparison to the traditional ITTP protocol.

One of the most recent grouping techniques was suggested by Abualhaj M. et al in 2020 [21]. Similar to the previous technique (CA-ITTP), the suggested grouping technique works over the ITTP protocol. The name of the suggested technique is payload shrinking and packets coalesce (PS-PC). The main objective of PS-PC technique is to lessen the wasted bandwidth resulted from the VoIP service. In order to do that, the PS-PC technique groups several VoIP packets in one IP preamble while adding a new runt preamble to each grouped VoIP packet. The runt preamble helps to extract and construct the original VoIP packet. Besides grouping the packets, the PS-PC technique compresses the VoIP packet payload based on a new novel algorithm. Together, packet grouping and payload compression have achieved a very high bandwidth saving. The PS-PC technique has been implemented and compared to the traditional technique (with no grouping neither compressing). The empirical results have shown that the suggested PS-PC technique outperforms the traditional technique in terms of preamble overhead and consumed bandwidth. Whereas, the preamble overhead and the consumed bandwidth have reduced by up to $25 \%$ and $51 \%$, respectively, in the tested cases. 
As we can see, VoIP packets grouping achieves a noticeable improvement of bandwidth saving. In this article, we suggest a new grouping technique that works with ITTP protocol. Unlike the previous techniques, the grouping technique that works over ITTP, the suggested technique performs the grouping process at the ITTP layer, not the IP layer, which achieves better bandwidth saving. The suggested technique is called ITTP Packets Grouping (ITTP-PG). The following section will discuss the suggested ITTP-PG technique in detail.

\section{SugGESTED ITTP-PG TECHNIQUE}

This section elaborates on the suggested ITTP-PG technique in detail. The ITTP-PG technique has the necessary components and performs the necessary steps to successfully fulfill better bandwidth improvements. The core of the suggested ITTP-PG technique is based on a key approach of improving the VoIP bandwidth utilization; which is reducing the preamble overhead $[18,20]$. As mentioned earlier, the suggested ITTP-PG technique intended to do so by group a plurality of VoIP packets in one preamble. Fig. 2 shows a VoIP network topology, in which the suggested ITTP-PG technique may be implemented and gives the maximal performance. The VoIP network topology in Fig. 2 assumes a company with several branches: branch $\mathrm{A}$, branch $\mathrm{B}$, branch $\mathrm{C}$, branch $\mathrm{D}$, etc. (e.g., a bank), with plenty of VoIP users in each branch. The VoIP user at each branch may make a call to any other branch at any time. The more the concurrent calls between any two branches (e.g., Branch A and Branch B) the better the performance of the suggested ITTP-PG technique, as we will discuss later in this section. The ITTP-PG technique is made up of two main entities. The first entity performs packets grouping (Pkt-G) and resides at the sender gateway. The second entity performs packets de-grouping (Pkt-DG) and resides at the receiver gateway. The following sections discuss the Pkt-G entity and Pkt-DG entity in detail.

\section{A. ITTP-PG Technique: Pkt-G Entity}

The Pkt-G entity resides at the sender side gateway. The main task of the Pkt-G entity is to group the VoIP packets from different client conversation sources and group them in one ITTP/IP preamble. The grouped packet must be intended to go to the same destination VoIP gateway. The process of packet grouping goes through several steps at the Pkt-G entity. The packets from different conversion sources are gathered in one buffer. Then, the packets are distributed to a different buffer based on their destination gateways. After that, the voice payload of the buffered packets is extracted and a runtpreamble is attached to each voice payload to form a runpacket. The runt-preamble will be discussed later in this section. Fig. 3 shows the format of the runt-packet. Subsequently, the resulting run-packets from each buffer, separately, are grouped in one ITT/IP preamble, which constitutes a giant-packet. Fig. 4 shows the format of the giant- packet. Finally, the giant-packets are transmitted to their destination gateways. Fig. 5 shows the Pkt-G entity at the sender gateway. Fig. 6 shows a flowchart of the internal process of the Pkt-G entity in the sender gateway.

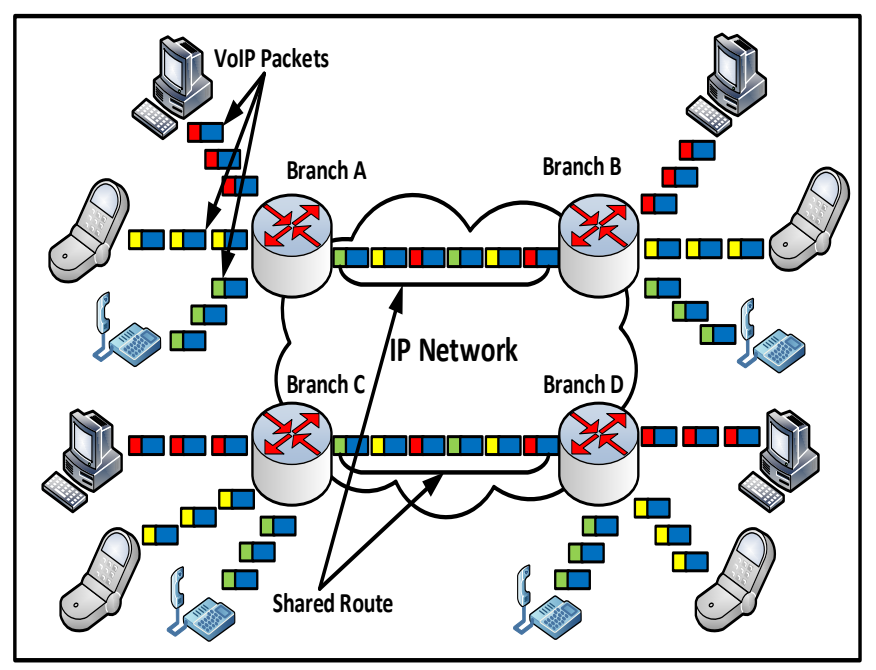

Fig. 2. ITTP-Mux Network Topology Scenario.

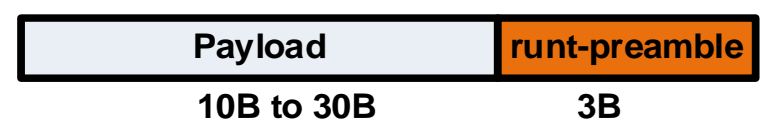

Fig. 3. Runt-Packet Format.

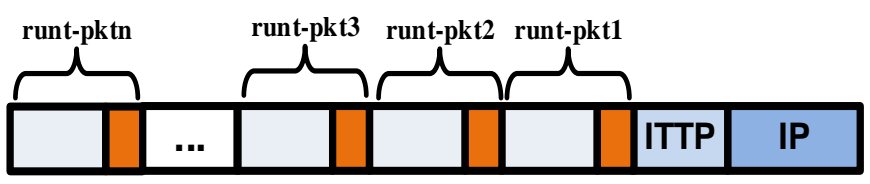

Fig. 4. Giant-Packet Format.

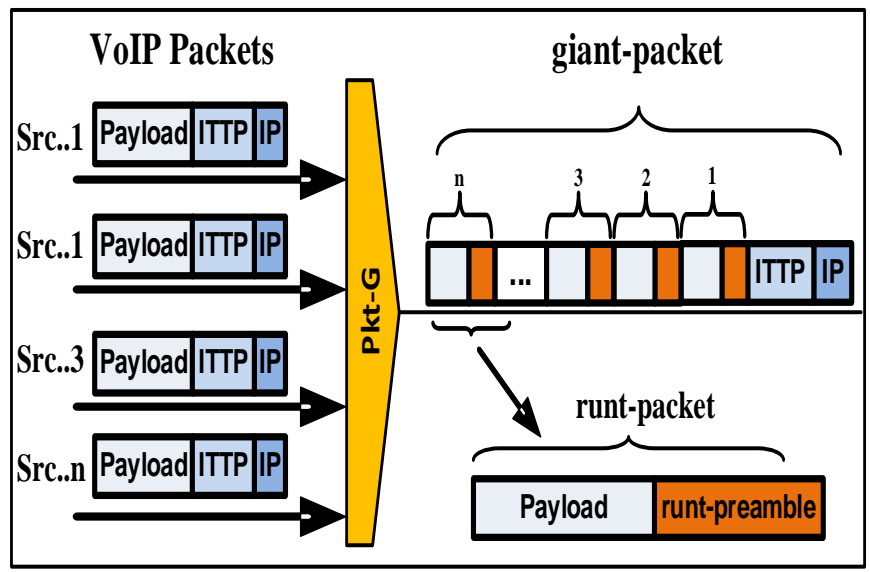

Fig. 5. ITTP-PG Technique: Pkt-G Entity. 


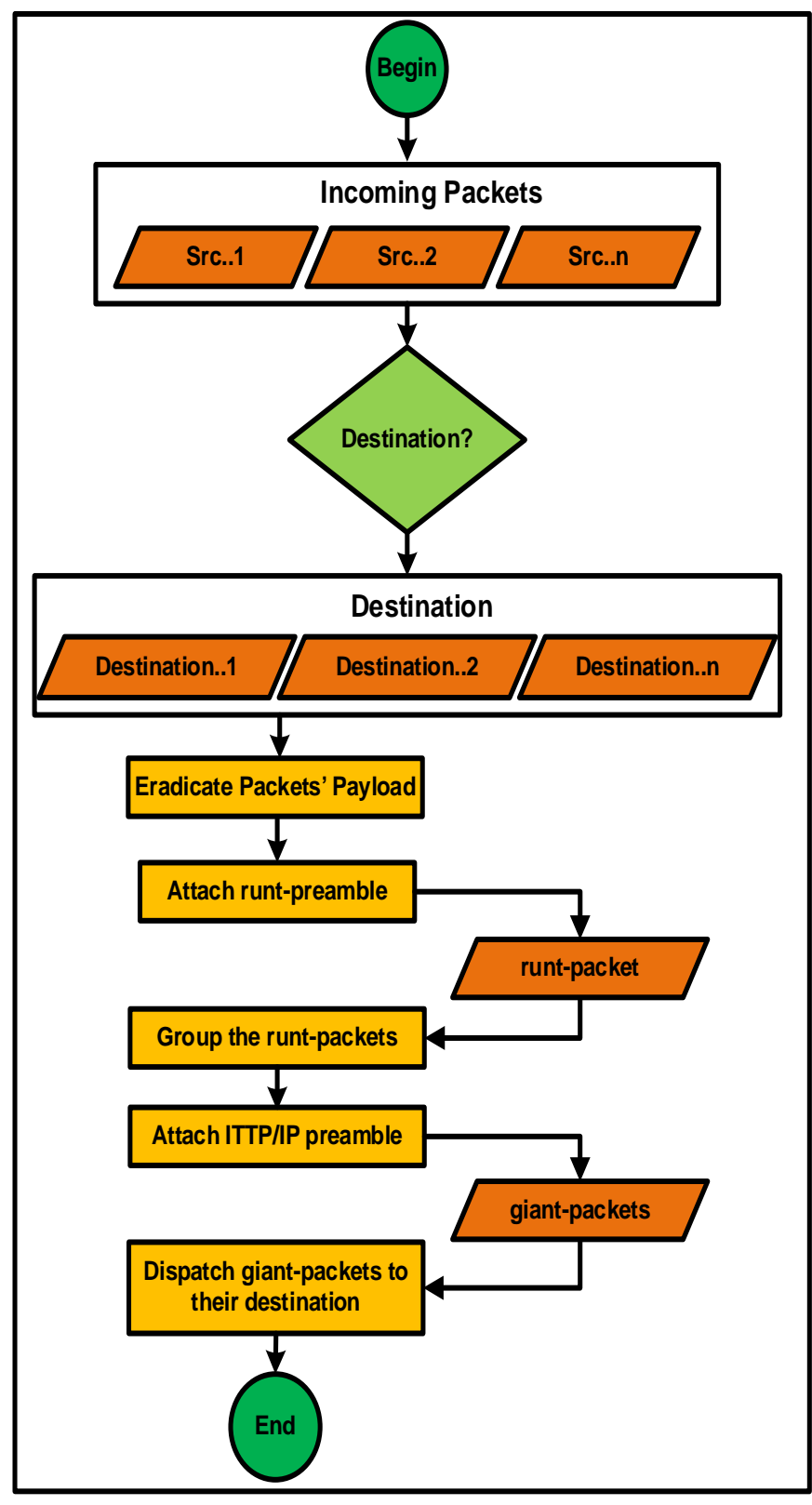

Fig. 6. Pkt-G Entity Operations.

\section{B. ITTP-PG Technique: Pkt-DG Entity}

The Pkt-DG entity resides at the receiver side gateway. The main task of the Pkt-DG entity is to de-group the giant-packets and restore the original VoIP packets. The process of packet de-grouping goes through several steps at the Pkt-DG entity. The incoming giant-packets are gathered in a single buffer by the Pkt-DG entity at the receiver gateway. Then, each giantpacket is de-grouped into runt-packets by inspecting the runtpreamble. After that, the voice frame is extracted by removing the runt-preamble from the runt-packet. Subsequently, the original packets are produced by adding ITTP/IP preamble to each voice frame, based on the information in the runtpreamble. Finally, the original packets are sent to their destinations. Fig. 7 shows the Pkt-DG entity in the receiver gateway. Fig. 8 shows a flowchart of the internal process of the Pkt-DG entity in the receiver gateway.

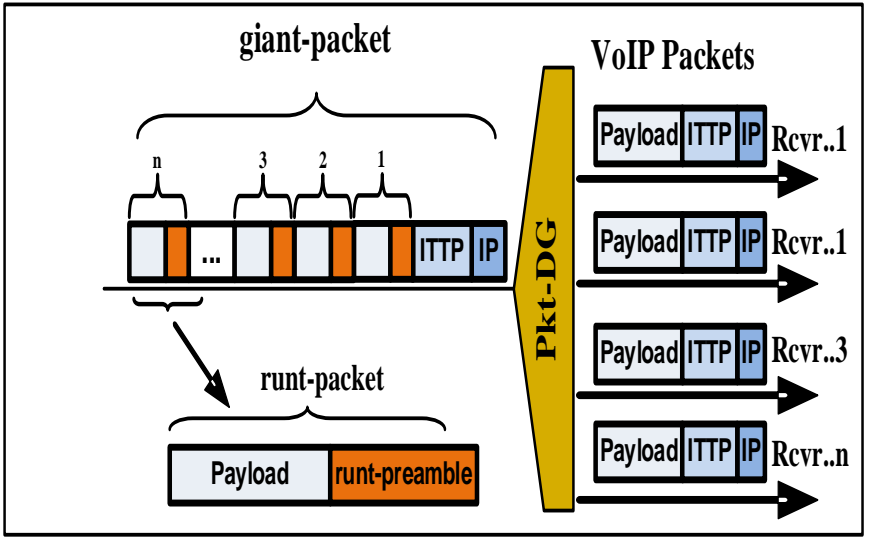

Fig. 7. ITTP-PG Technique: Pkt-DG Entity.

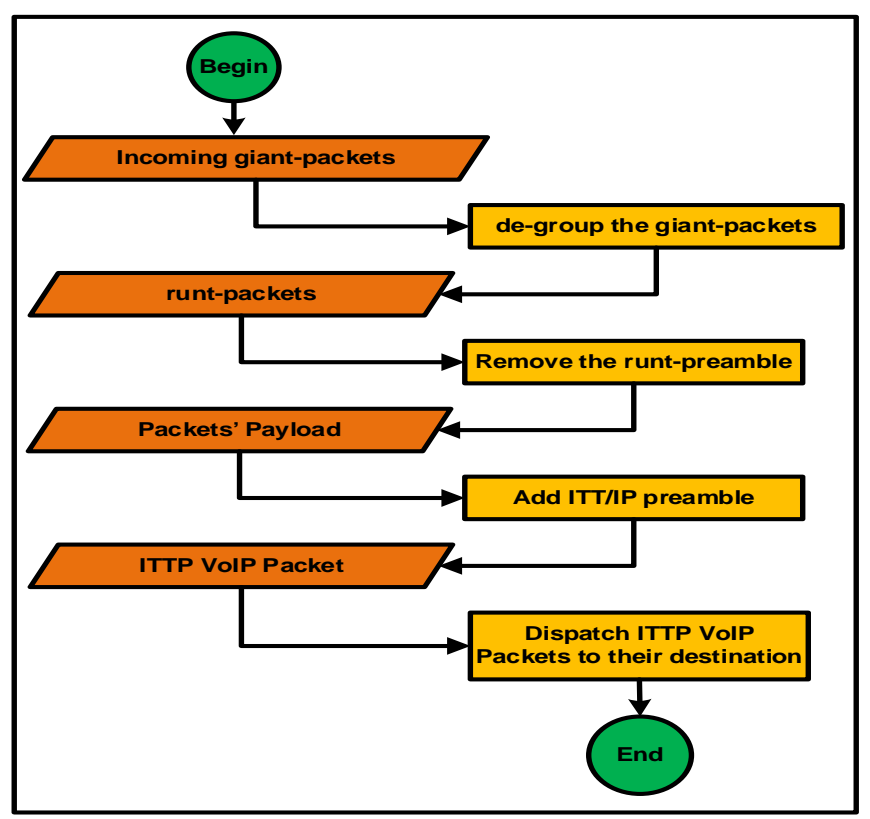

Fig. 8. Pkt-G Entity Operations.

\section{ITTP-PG Technique: Runt-Preamble}

The key goal of the suggested ITTP-PG technique is to eliminate the ITTP/IP preamble of each packet and group the voice frames of the packets directing to the same destination gateway into a single giant-packet of one ITTP/IP preamble. Before grouping these voice frames, a 3-byte runt-preamble is attached to each voice frame instead of the ITTP/IP preamble, which creates the run-packet. The Pkt-DG entity at the receiver side gateway uses this runt-preamble to separate the runtpackets within the giant-packet. The runt-preamble is composed of two fields namely a 1-byte conversation ID (CID) and 2-byte timestamp. Fig. 9 shows the runt-preamble format.

The timestamp is a key field in the ITTP protocol. The timestamp represents the number of milliseconds since the first data packet transmission of the call. The same value of the timestamp field in the ITTP protocol is saved in the timestamp field in the runt-preamble. The timestamp value is copied back to the original ITTP preamble at the receiver side by the PktDG entity. 


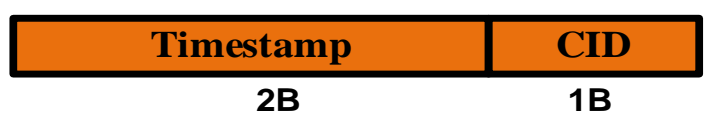

Fig. 9. Runt-Preamble Format.

The key use of the CID field is to find the destination address of the runt-packet, before being manipulated and grouped at the sender side. While building the giant-packet, the Pkt-G entity at the sender side gateway generates a unique CID for each runt-packet within a giant-packet. For each CID, a record is saved in a special table, called the address table, at the Pkt-G entity at the sender side. Each record in the address table contains the CID of the runt-packet and the original address (IP: Port Number) of the runt-packet before being grouped. A copy of the content of the address table is transmitted periodically to the Pkt-DG entity at the receiver side. Table II shows the address table. The size of the CID field can vary depend on the number of grouped runt-packets inside a giantpacket. Clearly, a 1-byte CID is enough to group 256 runtpackets inside one giant-packet. Obviously, replacing the 26byte ITT/IP preamble with the 3-byte runt-preamble will reduce the preamble overhead problem and saves the network bandwidth.

TABLE II. ADDRESS TABLE

\begin{tabular}{|l|l|l|l|l|}
\hline \multicolumn{2}{|l|}{ Pkt-G entity- Sender Side } & & \multicolumn{2}{l|}{ Pkt-DG entity- Receiver Side } \\
\cline { 1 - 1 } \cline { 1 - 1 } CID & IP address:port number & CID & IP address:port number \\
\hline 92 & $172.168 .0 .8: 5050$ & 92 & $172.168 .0 .8: 5050$ \\
\hline 20 & $172.168 .0 .9: 5051$ & 20 & $172.168 .0 .9: 5051$ \\
\hline 7 & $172.168 .0 .10: 5052$ & 7 & $172.168 .0 .10: 5052$ \\
\hline 15 & $172.168 .0 .11: 5055$ & 15 & $172.168 .0 .11: 5055$ \\
\hline
\end{tabular}

\section{ITTP-PG TECHNIQUE EVALUATION}

This section assesses the suggested ITTP-PG technique against the conventional ITTP protocol (without grouping) technique, which is called, for simplicity, C-ITTP. The effectiveness of the ITTP-PG technique against the C-ITTP technique is estimated in terms of bandwidth consumption, based on three primary elements. The first element is the number of concurrent VoIP calls (Call-N). Fig. 10 presents the Call-N of the ITTP-PG technique and the C-ITTP technique. The Call-N when using the ITTP-PG technique is greater than the Call-N when using C-ITTP. In addition, the difference in the Call-N increases when the available bandwidth increases.

The second element is the preamble overhead. The preamble overhead ratio is calculated by dividing the packet preamble size by the whole packet size. Fig. 11 presents the preamble overhead ratio of the ITTP-PG technique against the C-ITTP technique. The ITTP-PG technique achieved a significant reduction in preamble overhead against the C-ITTP technique.

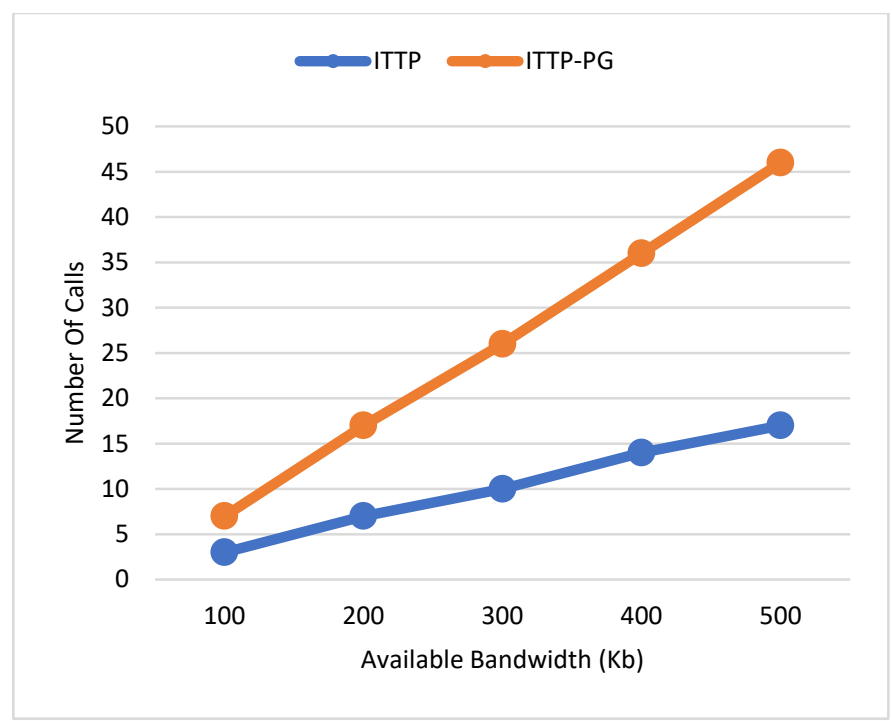

Fig. 10. Call-N of C-ITTP and ITTP-PG Techniques.

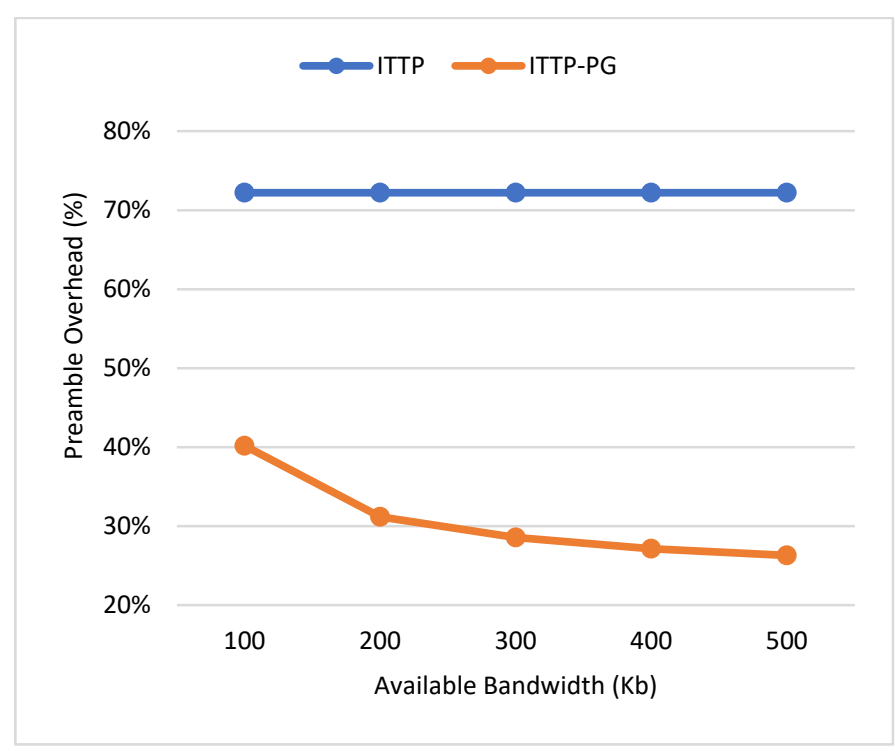

Fig. 11. Preamble Overhead Ratio.

The third element is the bandwidth usage. The bandwidth usage ratio is calculated by dividing the packet payload size by the whole packet size. Fig. 12 presents the bandwidth usage ratio of the ITTP-PG technique against the C-ITTP technique. The ITTP-PG technique achieved a significant enhancement in bandwidth usage against the C-ITTP technique. The enhancement of these three elements is because the C-ITTP technique attaches 26 bytes of a preambleto each payload while the ITTP-PG technique attaches only 3 bytes of a preamble to each payload and 20 bytes of IP preamble to the entire giantpacket. 


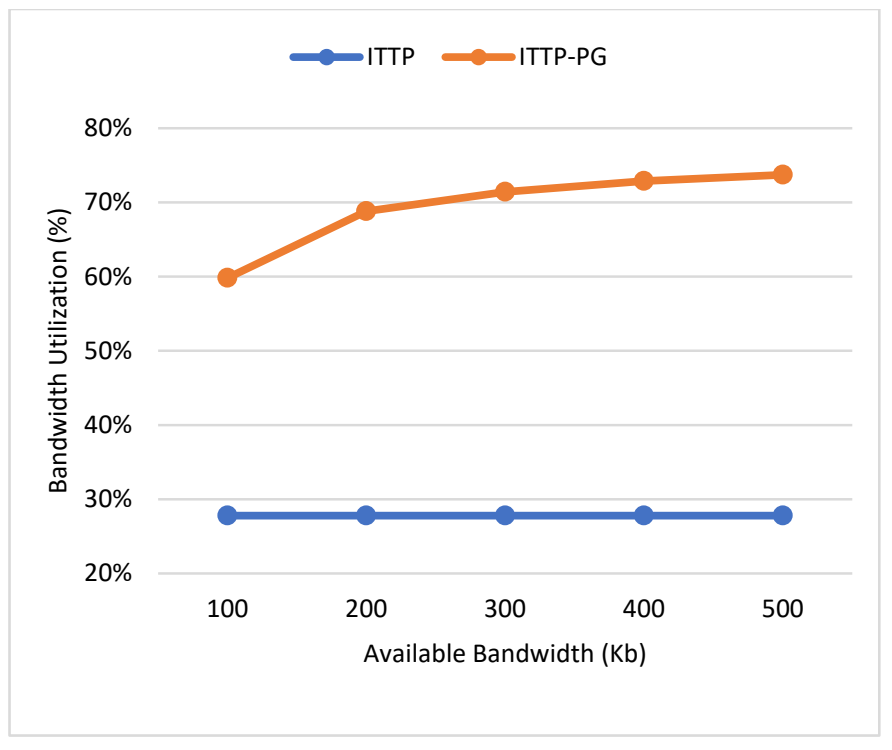

Fig. 12. Bandwidth usage Ratio.

\section{CONClUSiOnS}

VoIP appeared in the last decade as a new service in the telecommunications industry. VoIP service applications convey VoIP packets in short sizes, which increases preamble overhead of the packets and results in inefficient bandwidth employments. In this article, we suggested an efficient grouping technique called ITTP-PG. The ITTP-PG technique made up of the Pkt-G entity resides at the sender gateway and of Pkt-DG entity resides at the receiver gateway entities. The Pkt-G entity groups the VoIP packets in one ITTP/IP preamble. The Pkt-DG entity de-groups the giant-packets and restores the original VoIP packets. The performance of the ITTP-PG technique was assessed based on the number of concurrent VoIP calls, preamble overhead, and bandwidth usage. Based on these three elements, the ITTP-PG technique outperformed the C-ITTP technique. The three elements reflect bandwidth employments efficiency. In general, the simulation result showed that ITTP-PG improves bandwidth usage by up to $45.9 \%$, in comparison to the C-ITTP technique. In future, the proposed method will be implemented in real environment scenarios and investigated with the other comparable methods.

\section{REFERENCES}

[1] Gupta and A. Chaudhary, "A metaheuristic method to hide MP3 sound in JPEG image," Neural Computing and Applications, vol. 30(5), pp. 1611-1618, 2018.

[2] Q. Shambour, S. N. Alkhatib, M. M. Abualhaj, and Y. Alrabanah, "Effective voice frame shrinking method to enhance VoIP bandwidth exploitation,” International Journal of Advanced Computer Science and Applications (IJACSA), vol. 11(7), pp. 313-319, 2020.

[3] M. M. Abualhaj, Q. Y. Shambour, and A. H. Hussein, "Effective packet multiplexing method to improve bandwidth utilisation," International Journal of Computer Applications in Technology, vol. 63(4), pp. 327336, 2020.

[4] P. Christian https://blog.telegeography.com/voice-traffics-slumpcontinued-in-a-big-way-last-year, 2019.
[5] M. M. Abualhaj, S. N. Al-Khatib, M. Kolhar, A. Munther, and Y. Alraba'nah, "Effective voice frame pruning method to increase VoIP call capacity,” TEM Journal, vol. 9(1), pp. 48-54, 2020.

[6] C.Olariu, J.Fitzpatrick, Y. Ghamri-Doudane, and L. Murphy, "A delayaware packet prioritisation mechanism for voice over ip in wireless mesh networks," In 2016 IEEE wireless communications and networking conference, pp. 1-7, 2016.

[7] C.Vulkan, A. Rakos, Z. Vincze, and A. Drozdy, "Reducing overhead on voice traffic,” U.S. Patent No. 8,824,304. Washington, DC: U.S. Patent and Trademark Office, 2014.

[8] P. Fortuna and M. Ricardo, "Header compressed VoIP in IEEE 802.11," IEEE Wireless communications, vol. 16(3), pp. 69-75, 2009.

[9] M. M. Abualhaj, M. M. Al-Tahrawi, and S. N. Al-Khatib, "A new method to improve voice over ip (VoIP) bandwidth utilization over internet telephony transport protocol (ittp),” Proceedings of the 2019 8th International Conference on Software and Information Engineering, pp. 192-195, 2019.

[10] E. Charfi, L. Chaari, and L. Kamoun, "Joint urgency delay scheduler and adaptive aggregation technique in IEEE 802.11 n networks,” 2015 5th International Conference on Communications and Networking (COMNET), IEEE, pp. 1-6, 2015.

[11] H. Sathu and A. S. Mohib, "Performance comparison of VoIP codecs on multiple operating systems using IPv4 and IPv6," International Journal of e-Education, e-Business, e-Management and e-Learning, vol 2(2), pp. 122-125, 2012.

[12] S. Karapantazis and F.N. Pavlidou, "VoIP: a comprehensive survey on a promising technology," Computer Networks, vol. 53(12), pp. 20502090, 2009.

[13] M. M. Abualhaj, M. M. Al-Tahrawi, and S. N. Al-Khatib, "Performance evaluation of voip systems in cloud computing," Journal of Engineering Science and Technology, vol. 14(3), pp. 1398-1405, 2019.

[14] R. Safoine, S. Mounir, and A. Farchi, "Comparative study on DOS attacks detection techniques in SIP-based VoIP networks," 2018 6th International Conference on Multimedia Computing and Systems (ICMCS), IEEE, pp. 1-5, 2018.

[15] M. Spencer, B.Capouch, E.Guy, F.Miller, and K. Shumard, Iax: Interasterisk exchange version 2, Internet Request for Comments, 2010.

[16] N. Gupta, N. Kumar, and H. Kumar, "Comparative analysis of voice codecs over different environment scenarios in VoIP," 2018 Second International Conference on Intelligent Computing and Control Systems (ICICCS), IEEE, pp. 540-544, 2018.

[17] A. K. Chilli, K. R. Prasanna Kumar, H. A. Murthy, and C. C. Sekhar, "Approaches to codec independent speaker identification in VoIP speech,” 2018 Twenty Fourth National Conference on Communications (NCC), IEEE, pp. 1-5, 2018.

[18] Y. Nomura, K. Mori, K. Naito, and H. Kobayashi, "High efficient packet aggregation scheme for multi-rate and VoIP packet transmissions in next generation MU-MIMO WLANs," 2014 International Conference on Advanced Technologies for Communications (ATC 2014), IEEE, pp. 517-521, 2014.

[19] P. H. Azevêdo Filho, M. F. Caetano, and J. L. Bordim, "A packet aggregation mechanism for real time applications over wireless networks," International Journal of Networking and Computing, Vol 2(1), pp. 18-40, 2012.

[20] M. M. Abualhaj, "CA-ITTP: An efficient method to aggregate voip packets over ittp protocol," International Journal of Innovative Computing, Information and Control (IJICIC), vol. 15(3), pp. 10671077, 2019.

[21] M. M. Abualhaj, S. N. Al-Khatib, and Q. Y. Shambour, "PS-PC: An Effective Method to Improve VoIP Technology Bandwidth Utilization over ITTP Protocol,” Cybernetics and Information Technologies, vol. 20(3), pp. 147-158, 2020. 